\title{
Games with(out) Frontiers: toward an integrated science of human cognition
}

\section{Bernhard Hommel* and Lorenza S. Colzato}

Cognitive Psychology Unit, Leiden Institute for Brain and Cognition, Leiden University, Leiden, Netherlands

*Correspondence: hommel@fsw.leidenuniv.nl

Starting up a new journal is an excellent occasion to reflect not only on the directions into which the targeted field is currently heading but also on the remaining challenges to be met and the shortcomings to be overcome. We will do so by putting research on human cognition (the field that we as the new specialty editors of Frontiers in Cognition represent) into a broader perspective and consider, among other things, the central role this research plays, or at least has the potential to play, in connecting other scientific areas and disciplines, as well as the social and infrastructural changes that will be necessary to successfully tackle the challenges that remain on our way to a truly integrative science of human cognition.

\section{EXPLOITING NEUROSCIENCE}

When one of us (Bernhard Hommel) first met John Gabrieli in the early 1990s on a very interdisciplinary and (thus) very exciting meeting in Berlin, he was still skeptical about the use of the back then increasingly popular neuroimaging methods for the functional understanding of cognitive processes. "But however you think about it", Gabrieli replied, "you got to admit that the cognitive neurosciences have made cognitive psychology so much more interesting". And this is certainly true: neuroscientific observations have sparked numerous cognitive theories, approaches, and discoveries, just think of research on the binding problem and the notorious mirror neurons. Hence, the cognitive neurosciences have strongly affected cognitive psychology but they also keep challenging it and call for, so we argue, a reconsideration of how cognitive psychologists organize and carry out their research, both technically and socially.

The great success of the cognitive neurosciences has already begun to shape the way psychologists ask their research questions, at least if they make use of modern neuroscientific techniques. Instead of asking the traditional question of how a particular cognitive mechanism works, increasing numbers of studies are targeting the "neural correlates" of these mechanisms or of the phenomenon under study. The outcome of such endeavors is pointers to particular brain areas, as if this would tell us anything about how the mechanism works or what algorithm it applies. It is certainly true that knowing about the brain areas involved can provide a first step toward the investigation of a mechanism, to be followed by the study of the interactions between such areas, and the mapping of these interactions to particular cognitive algorithms. But these following steps rarely take place and many psychologists are ready to accept the outcome of the localization enterprise as an important observation in itself. Moreover, studies that are linking a particular brain area to a particular cognitive process commonly neglect the fact that the same area is also involved in numerous other, often unrelated types of processes, and attempts to explain why that is are extremely rare as well. Hence, the cognitive neurosciences do not only provide us with interesting new observations but their success and attraction for psychological students also tends to push psychological theorizing toward a reductionist perspective that "explains" cognitive mechanisms by equating them with the brain structures that might be hosting them.

It will be crucial for the future of cognitive psychology and the cognitive sciences in general how we deal with this challenge. One possibility is self-chosen splendid isolation and, indeed, numerous cognitive psychologists are developing an often emotionally colored antipathetic attitude against the neurosciences and their methods. Unfortunately, this trend is facilitated by the fact that we are facing an increasing number of symposia and conferences to choose from at times of financial crisis and tight travel budgets, so that many of us are to decide whether to go to more "behavioral" or more "neuroscientific" meetings. As a consequence, meetings with a traditionally strong behavioral emphasis are losing sight of the cognitive neurosciences, while hardcore neuroscientific meetings are no longer visited by many colleagues with a strong behavioral and theoretical background.

The other alternative takes much more effort, money, and possibly even changes in our social behavior. In principle, cognitive psychology has a lot to offer to the cognitive neurosciences and many discoveries in this area would not have been possible without the systematic development of tasks, paradigms, and analytical methods of cognitive psychologists. However, the steadily growing neuroscientific machinery poses very high demands in terms of technical, methodological, and analytical skills on the individuals using it, and only few, if any single researcher could ever master even the main techniques (say, EEG, fMRI, TMS, and PET) to a satisfying degree. Depending on background, abilities, age, money, and teaching load, some individuals may be successful working themselves into one of these techniques or perhaps two, but even this individual achievement would be insufficient to reach what should be the ideal situation: to flexibly pick the method that is suited best to address a particular research question.

Unfortunately, these enormous challenges tend to make the first alternative much more attractive to quite a few. As a consequence, there is a real danger for a schism between theoretically guided, functionally oriented (but mainly behavioral) cognitive psychology on the one hand and an increasingly atheoretical, data-driven cognitive neuroscience on the other. In our view, it will be crucial for the future success of cognitive psychology to tackle this problem. No doubt, this will need aggressive efforts and, presumably, substantial changes in social networking and the infrastructural requirements for conducting and funding research. Given the increasing technical demands posed by research techniques (even if these will be alleviated by software development to some degree), flexibly constructed social 
networks of researchers with very different backgrounds, expertise, and interests will be necessary - networks that will be tailored to the research question at hand and, thus, likely to change from one question to the other. To some degree, this is trivial to say and reflects what successful research teams are practicing already. However, it is also true that the ways research is academically organized and funded, and individual researchers are evaluated, often do not fit with the requirements of this research style (e.g., by favoring competition over cooperation). Moreover, exposing oneself to interdisciplinary research environments and networks has very unfortunate psychological side effects: researchers who have worked very hard to reach a particular level of expertise and self-confidence are now thrown back to a beginner's level in several respects. This is difficult to bear without having been trained to overcome the resulting frustrations, and to acquire an interdisciplinary attitude and the necessary social skills from the start. This attitude and these skills can only be acquired in respective interdisciplinary environments, BA and MA programs, and research schools - which are still hard to find.

Apart from these more administrative and political challenges, cognitive psychologists need to assume a more assertive, self-confident attitude with respect to the cognitive neurosciences. Here the challenge will be to exploit neuroscientific knowledge and techniques without giving up the very high theoretical standards that cognitive psychology has developed and that it can be proud of. This will no doubt be difficult and require the acquisition of at least basic knowledge about neuroimaging and related techniques, but might become easier to manage though expansive social networking. In any case, however, it will be important that cognitive psychologists expand their methodological toolbox and raise their voice more distinctly in debates regarding the implications of neuroscientific evidence for the functional understanding of cognitive mechanisms and the brain processes underlying them.

\section{BRIDGING HUMANITIES AND NATURAL SCIENCES}

The recent decades have shifted the focus from the humanities to the natural sciences, so that, for just one of many examples, the general public is now more interested in the brain areas involved in moral decisionmaking than in the development, justification, and societal implementation of the moral guidelines on which these decisions are being based. This has put the academic organization of the humanities under substantial economic pressure (as witnessed by the cancellation of many chairs and study programs in that area) and sometimes triggered discussions about their societal function and use. This is not the place for a discussion of that issue but we would like to emphasize an interesting and, from the perspective of cognitive psychology, perhaps very fruitful side effect of this process.

Being under pressure, many disciplines in the humanities are opening up in terms of scope and approach to their topics. Given the noticeable success of the natural sciences, it is not surprising that new ways are considered to bring humanities and natural sciences into closer contact and to create overlap wherever possible-some countries (e.g., Germany) have even launched dedicated funding programs to achieve that. This provides unique opportunities for cognitive psychology, as it lies right at the interface between the humanities and the natural sciences. Other disciplines and subdisciplines have made ample use of this particular position already, and freely borrowed cognitive concepts, models, and paradigms to become more empirical, experimental, and in part biological. There are many examples, ranging from the development of the social cognition approach in social psychology (cf., Hommel, 2006a) over the search for the neural correlates of consciousness to neuroeconomics. Even though the resulting interdisciplinary endeavors are exciting and highly promising, cognitive psychologists could have played a more active and proactive role in these and other developments, and could do so in the future. Many more bridges are needed indeed, just think of the cognitive implications of religion, literature, ethics, and more. It seems important to us that cognitive psychologists become more expansive with respect to the borders of their (sub)discipline and make more active use of the fact that they are interfacing with almost any area covered by the humanities. Their experimental expertise and often broad theoretical knowledge seems much more precious and have more potential for bridging other disciplines than many of us are aware of. Fulfilling his potential is likely to strongly increase the breadth and validity of our theories and models and provide interesting avenues for testing them.

\section{INVESTIGATING PROCESSES, NOT PARADIGMS}

Experimental paradigms are at the core of our trade. It is to a large extent paradigms that have made cognitive psychology so successful - well-understood tasks that have been carefully developed and systematically extended. This knowledge about paradigms and their theoretical implications is what neighboring areas and disciplines are after, what has been adopted from the clinical sciences in the search for process-pure measures of particular cognitive impairments and by the social sciences to make abstract theoretical concepts empirically fruitful. Paradigms are also one of the main contributions of cognitive psychology to the emerging cognitive sciences, and most of the important contributions to the functional understanding of human cognition from neuroimaging research would have been impossible without the employment of well-developed cognitive paradigms.

And yet, cognitive psychology tends to overdo with respect to the enthusiasm for paradigms, which often stands in the way of theoretical progress in a wider sense. We devote whole research careers to one single experimental task or effect that often has very little if any ecological validity. There are obvious advantages of this strategy. Becoming truly proficient in applying and making optimal use of a given paradigm takes a lot of time and effort, which should not be wasted by jumping from one paradigm to another. Moreover, sticking to one paradigm or effect makes it much easier to develop a unique research profile and acquire a solid scientific standing, and it is often tasks and effects that bring together researchers in special issues and lively scientific symposia. And yet, the resulting over-emphasis on paradigms and effects has a number of serious consequences that we need to overcome on our way to a truly integrated approach to human cognition.

Paradigm-driven research necessarily aims at understanding whatever effects the paradigm produces. This has many disadvantages. For one, a given paradigm might be an excellent, close to process-pure 
measure of one cognitive process or mechanism but it is likely to be a lousy measure of numerous other processes or mechanisms. If so, why would one even try to understand the role of the latter in this particular task rather than using another, better-suited task to assess those other processes? And yet, given our fixation on tasks and effects, many models we develop are trying to capture all the processes involved therein and, indeed, we as reviewers and editors would insist that authors include all these processes in their model. Would it not be better to follow the exact opposite strategy? That is, to try to capture one given process across as many paradigms and effects as possible, thereby explicitly ignoring the fact that these paradigms and effects are not equivalent with respect to a number of other processes. And to try to evaluate our favorite paradigms and effects much more in terms of how well they capture one given process. Of course, this strategy would by no means be foolproof and likely to generate a new kind of "specialist" that is narrowminded not in terms of tasks but in terms of processes. And yet, it seems essential to us that we at least try to reduce our present fixation on paradigms and effects. One of several benefits of such a strategy is that we could be much more certain that our research topic has external validity. Indeed, it is much easier to explain how important it is to investigate, say, the human will or decision-making under uncertainty than to try relating the task-switching paradigm or the Stroop effect to the real world.

The lesson to be learned, we think, is to reconsider our paradigms and effects as what they are: means to an end but not ends by themselves. The true goal of an integrated cognitive science is the understanding and modeling of cognitive mechanisms (measured as purely as possible) and the understanding of psychological phenomena, that ideally are either taken from, or are at least modeled after behavior that can be found outside of our labs.

\section{INTEGRATING MODELING AND EXPERIMENTATION}

A discipline's level of maturity is often measured by the degree to which the phenomena it addresses can be captured by mathematical models. Even though one can argue whether this is a particularly important or even the crucial criterion, it would certainly advance our discipline if empirical research, theoretical reasoning, and computational modeling would integrate better. And yet, it seems fair to say that computational models do not play a particularly dominant role in most areas of cognitive psychology. How can that be improved?

There are likely to be numerous answers, and many will have to do with the types of models, the ways they are tested, and how they are communicated, but there is one answer that we would like to emphasize. As developed in some more detail elsewhere (Hommel, 2006b), computational models are much more successful and influential if they come with new and unexpected empirical data, that is, if model construction and model testing go hand in hand and if the data do not only serve to justify the model. Instead, models that merely redescribe old or replicated data from highly particular paradigms commonly fail to impress experimental researchers sufficiently to adopt and work with them.

However, being a good modeler and a good, broadly experienced experimentalist at the same time is quite a challenge that most of us are not up to, a problem that is similar to our cognitive and time restrictions with regard to the acquisition of neuroscientific methods, as discussed above. This is why we consider it essential for computational modelers to team up with experimentalists, probably even from diverse areas, to get the intended cycle from modeling to testing to model modification to testing etc. really going and for making it really interesting. What is also likely to help is to provide interested colleagues with the software tools necessary to generate model predictions and to test the model, as in the ACT-R community (act-r.psy.cmu.edu). Only then, we think, would it be possible to achieve what most models promise but actually fail to achieve: the generation of really new and relevant, but unexpected and counterintuitive predictions that can be demonstrated to stand empirical test, ideally across different tasks and paradigms. Only if that level could be reached at a broader scale, computational models would impact our theorizing to a degree that goes beyond mere existence proofs or the demonstration of computational principles (which of course will also remain important).

\section{CREATING SUITABLE RESEARCH CONTEXTS}

Progress in science is often associated with competition, and competition is undeniably a particularly strong motivating factor. And yet, there are reasons to believe that overemphasizing this factor may seriously limit progress in science. Performing research is increasingly complex and complicated, especially if one is trying to go beyond single effects and paradigms but to integrate multiple ideas and concepts. To deal with this complexity, we have argued, will often require teaming up with other colleagues, sometimes with different types of expertise and background, and even from different disciplines. To do so effectively presupposes solid social skills. Even though that might be trivial to say we don't think it is. Many of us grew up in academic systems that comprised of surprisingly many, commonly male "specialists" with surprisingly few skills and motivation to communicate and to spark enthusiasm for their research topics in others. This attitude may have sufficed for a scientific career in the past, but we suspect that decidedly stronger emphasis on skills in communication and social networking will be necessary to advance our discipline in the future.

Fortunately, there are at least two factors our discipline has benefited from in this respect. First, the development and widespread availability of software packages for the easy-to-access programming of psychological experiments has opened the door for increasing amounts of non-nerdy, socially proficient cognitive students and increased the ratio of female to male students in cognitive psychology. Second, the increasing amount of interaction between cognitive psychology and the cognitive neurosciences has attracted many students - many more students than traditional cognitive psychology and students with much more diverse backgrounds in scientific perspective. This greater diversity is likely to make discussions livelier and increase our tolerance and appreciation for seemingly weird outof-the-box ideas.

Fully exploiting this interdisciplinary attitude and the creative potential it provides requires strong efforts. For one, we need to counteract the centrifugal forces that are driving behavioral cognitive psychology and the cognitive neurosciences apart. Among other things, this means that 
we need to succeed in keeping or creating interesting scientific conferences or other types of meetings that are attractive for and thus visited by both behavioral and neuroscientific researchers. It also means that more interdisciplinary study programs and graduate schools will need to be launched, including programs that integrate psychological subdisciplines. But we also need to reconsider the reward systems handled by universities and funding agencies to see whether more could be done to facilitate collaboration and flexible networking.

\section{GAMES WITHOUT FRONTIERS}

To conclude, the recent developments in and around our field have the potential to bring us much closer to a well-integrated science of human cognition. Fulfilling this potential, so we have argued, will be much easier if we are strongly expanding or even doing away with at least four types of frontiers:

- Methodological frontiers, by including neuroscientific techniques and machinery in our experimental toolbox - not to trade our functional theories for neuroanatomical or neurophysiological hypotheses but to have more instruments at our disposal to test them.
- Paradigmatic frontiers, by tracing interesting cognitive mechanisms across various experimental paradigms and by making models focusing on these mechanisms but not on the paradigms assessing them.

- Disciplinary frontiers, by getting in closer touch to neighboring psychological subdisciplines and other disciplines, so to make productive use of our unique position as an interface between the humanities and the natural sciences.

- Personal frontiers, by engaging more in social networking and creating flexible, quickly changing research groups that cover a broad range of expertise and theoretical background.

Sure enough, tackling these challenges requires activities that go way beyond the mission of a scientific journal, even if Frontiers' interactive editorial style does provide new opportunities in this context. Nevertheless, we will do what we can in order to facilitate these processes, be it in the form of the editorial policy, which will emphasize innovation and courage, interdisciplinarity and theoretical breadth, or in the form of special issues that, in terms of topic or style, will help to turn our science from Newell's (1973) game of 20 questions with nature that we can't win into a cumulative science that provides us with an increasingly comprehensive understanding of human cognition and action.

\section{REFERENCES}

Hommel, B. (2006a). Bridging social and cognitive psychology? In Bridging Social Psychology: The Benefits of Transdisciplinary Approaches, P. A. M. Van Lange, ed. (Hillsdale, NJ, Erlbaum), pp. 167-172.

Hommel, B. (2006b). On the social psychology of modeling. Neural Netw. 19, 1455-1457.

Newell, A. (1973). You can't play 20 questions with nature and win: projective comments on the papers of this symposium. In Visual Information Processing, W. G. Chase, ed. (New York, Academic Press), pp. 283-308.

Received: 28 January 2010; accepted: 03 February 2010; published online: 08 March 2010.

Citation: Hommel B and Colzato LS (2010) Games with(out) Frontiers: toward an integrated science of human cognition. Front. Psychology 1:2. doi: 10.3389/ fpsyg.2010.00002

This article was submitted to Frontiers in Cognition a specialty of Frontiers in Psychology.

Copyright $\odot 2010$ Hommel and Colzato. This is an openaccess article subject to an exclusive license agreement between the authors and the Frontiers Research Foundation, which permits unrestricted use, distribution, and reproduction in any medium, provided the original authors and source are credited. 